\title{
G -factors in LaAlO3/SrTiO3 quantum dots
}

Bjørlig, Anders V.; Carrad, Damon J.; Prawiroatmodjo, Guenevere E.D.K.; Von Soosten, Merlin; Gan, Yulin; Chen, Yunzhong; Pryds, Nini; Paaske, Jens; Jespersen, Thomas S.

Published in:

Physical Review Materials

Link to article, DOI:

10.1103/PhysRevMaterials.4.122001

Publication date:

2020

Document Version

Publisher's PDF, also known as Version of record

Link back to DTU Orbit

Citation $(A P A)$ :

Bjørlig, A. V., Carrad, D. J., Prawiroatmodjo, G. E. D. K., Von Soosten, M., Gan, Y., Chen, Y., Pryds, N., Paaske, J., \& Jespersen, T. S. (2020). G -factors in LaAlO $/ \mathrm{SrTiO}$ quantum dots. Physical Review Materials, 4(12), [122001]. https://doi.org/10.1103/PhysRevMaterias.4.122001

\section{General rights}

Copyright and moral rights for the publications made accessible in the public portal are retained by the authors and/or other copyright owners and it is a condition of accessing publications that users recognise and abide by the legal requirements associated with these rights.

- Users may download and print one copy of any publication from the public portal for the purpose of private study or research.

- You may not further distribute the material or use it for any profit-making activity or commercial gain

- You may freely distribute the URL identifying the publication in the public portal 
PHYSICAL REVIEW MATERIALS 4, 122001(R) (2020)

Rapid Communications

\title{
$g$-factors in $\mathrm{LaAlO}_{3} / \mathrm{SrTiO}_{3}$ quantum dots
}

\author{
Anders V. Bjørlig, ${ }^{1}$ Damon J. Carrad $\odot,{ }^{1}$ Guenevere E. D. K. Prawiroatmodjo $\odot,{ }^{1}$ Merlin von Soosten, ${ }^{1,2}$ Yulin Gan $\odot,{ }^{2}$ \\ Yunzhong Chen, ${ }^{2}$ Nini Pryds, ${ }^{2}$ Jens Paaske, ${ }^{1}$ and Thomas S. Jespersen ${ }^{1}{ }^{1, *}$ \\ ${ }^{1}$ Center for Quantum Devices, Niels Bohr Institute, University of Copenhagen, Universitetsparken 5, 2100 Copenhagen, Denmark \\ ${ }^{2}$ Department of Energy Conversion and Storage, Technical University of Denmark, Ris $\phi$ Campus, 4000 Roskilde, Denmark
}

(Received 28 July 2020; accepted 13 November 2020; published 3 December 2020)

\begin{abstract}
We investigate the $g$-factors of individual electron states in gate-defined quantum dots fabricated from $\mathrm{LaAlO}_{3} / \mathrm{SrTiO}_{3}$ heterostructures. We consider both the case of effective positive charging energy $(U>0)$ where single electrons are added upon increasing the local gate voltage, and the case of $U<0$ where electron pairing is observed. The $g$-factors are extracted from the field dependence of the quantum dot addition spectrum. Tunnel couplings and confinement are tunable by the gate voltages and in the regime of weakest coupling, we find $g$-factors close to 2 due to quenching of the orbital magnetic moment. For stronger coupling, $g$-factors are anisotropic and exhibit values up to $\sim 4.5$ in the out-of-plane orientation. We further show examples of the sequential addition of electrons with the same spin as a consequence of exchange interactions.
\end{abstract}

DOI: 10.1103/PhysRevMaterials.4.122001

The spin properties of electrons confined to quantum dots (QD) have received significant attention due to their fundamental importance and the possible use as prototypical two-level systems for quantum information processing [1,2]. The spin decoherence in quantum dots is strongly influenced by the host material which sets the strength of hyperfine coupling to nuclei spins and of the spin-orbit interaction which couples the QD spin to lattice vibrations and charge fluctuations. In addition, the material platform also influences the experimental possibilities for confining, reading out, and controlling QD spins. For example, the canonical GaAs-based two-dimensional electron gases (2DEGs) allow accurate electrostatic control by local gates, and single-shot readout of electron spins has been demonstrated by spin-blockade experiments and high-frequency charge sensing [3], while spin control has been achieved by virtue of local magnetic fields [4], engineered field gradients [5], and the spin-orbit interaction (SOI) $[6,7]$.

The electron systems appearing in $\mathrm{SrTiO}_{3}$ (STO)-based heterostructures [8] present qualitatively new opportunities for quantum devices. On one hand, they share with semiconductors the key feature of being susceptible to electrostatic gating $[9,10]$ and thus allow the realization of devices using well-established gating concepts and fabrication using conventional semiconductor processing techniques [11-18]. However, in addition the oxides provide a range of functionalities unavailable in conventional semiconductors, making STO-based electron systems particularly interesting for hybrid quantum devices. These functionalities include intrinsic gate-tunable magnetic [19] and superconducting [20] phases, density-dependent spin-orbit interactions, as well as effectively attractive interactions leading to electron pairing in mesoscopic devices without superconductivity [15].

\footnotetext{
*tsand@nbi.ku.dk
}

The fundamental quantity characterizing the coupling of spin to an external magnetic field is the effective $g$-factor $g_{\text {e }}$ which parametrizes the magnitude of the splitting of spin pairs in a magnetic field. In bulk conventional semiconductors, deviations of the conduction band $g$-factor from $g_{0}=2$ are caused by spin-orbit-induced mixing between the conduction and valence bands, leading to a dependence on the band gap $E_{\mathrm{g}}$ [21]: $g_{\mathrm{e}}=2-2 E_{\mathrm{P}} \Delta_{\mathrm{SO}} /\left[3 E_{\mathrm{g}}\left(E_{\mathrm{g}}+\Delta_{\mathrm{SO}}\right)\right]$, where $\Delta_{\mathrm{SO}}$ is spin-orbit strength and $E_{\mathrm{P}}$ is a constant band overlap parameter. The situation differs for $\mathrm{LaAlO}_{3} / \mathrm{SrTiO}_{3}$ (LAO/STO) heterostructures where electrons populate the STO $t_{2 g}$ bands: On one hand, deviation of $g_{\mathrm{e}}$ from $g_{0}$ due to valence band mixing is strongly suppressed compared to semiconductors by the large band gap $\left(E_{\mathrm{g}}^{\mathrm{STO}} \sim 3 \mathrm{eV}\right)$. On the other hand, however, the $d_{\mathrm{xy}}$ band couples via the spin-orbit interaction to the $d_{\mathrm{xz}}$ and $d_{\mathrm{yz}}$ bands, which are split from the $d_{\mathrm{xy}}$ band by only a small energy, $<40 \mathrm{meV}$, due to the out-of-plane confinement [22,23]. This splitting decreases with increasing density towards the Lifshitz transition [23] and thus $g_{\mathrm{e}}$ deviations may increase with density; opposite of the semiconductor case. Such a dependence was observed in Ref. [24] with low-temperature $g_{\mathrm{e}}$ extracted from weak antilocalization measurements, showing an increase from 0.5 to 2 towards the Lifshitz transition. For bulk STO, $g_{\mathrm{e}} \approx 2$ was found from electron spin resonance and values of up to 5 were reported from analysis of high-field Shubnikov-de Haas ( $\mathrm{SdH})$ oscillations [25].

In nanostructures, the deviations of $g_{\mathrm{e}}$ are suppressed further due to the finite level spacing increasing the effective gap, while in addition, $g_{\mathrm{e}}$ is influenced by the effects of SOI, lower symmetry, and size-dependent quenching of spin-correlated orbital magnetic moments [26-28]. The consequence is a strong dependence on the electrostatic confinement and the spatial distribution of individual wave functions. Compared to semiconductors, the large effective mass of STO, $m_{\mathrm{STO}} \sim 1 \mathrm{~m}_{\mathrm{e}}$ $\left(m_{\mathrm{GaAs}}=0.067 m_{\mathrm{e}}\right)$ reduces the influence of the finite level spacing, however, the quenching of orbital currents is still 

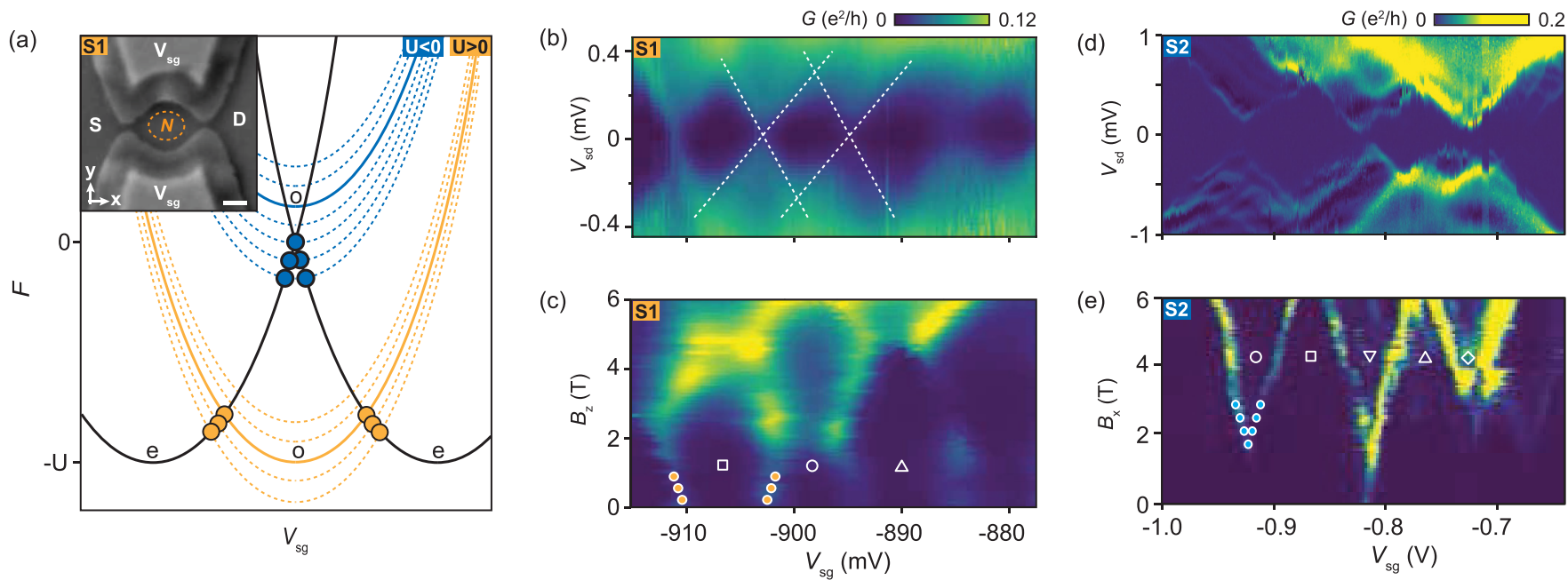

FIG. 1. (a) Free energy as a function of gate voltage for three successive charge states in the case of $U>0$ (orange curves) and $U<0$ (blue curves). Solid (dashed) lines correspond to $B=0(B>0)$. The odd- $N$ parabolas exhibit Zeemann splitting and the intersection points between even and odd charge states (circles), where zero-bias transport is allowed, shift linearly along $V_{\mathrm{sg}}$ (circles). The inset shows a top view scanning electron microscopy image of device $\mathrm{S} 1$. The scale bar is $100 \mathrm{~nm}$. (b), (d) $G \mathrm{vs} V_{\mathrm{sg}}$ and $V_{\mathrm{sd}}$ for samples $\mathrm{S} 1$ and $\mathrm{S} 2$, respectively. Dashed lines in (b) indicate the Coulomb blockade diamonds. (c), (e) Conductance vs $V_{\mathrm{sg}}$ and $B$ for $U>0$ (S1) and $U<0$ (S2), respectively. The circles indicate the linear shift of a pair of Coulomb peaks [cf. (a)].

expected to push $g_{\mathrm{e}}$ towards $g_{0}$ upon increasing the quantum dot confinement. So far, however, the spin properties of STObased QDs have not been systematically explored.

Experimentally, only a few reports of the $g$-factor in LAO/STO nanostructures exist: In Ref. [15], $g_{\mathrm{e}}=1.2$ was found for a QD exhibiting electron pairing, and values of $\sim 0.6$ were reported from measurements of the subband splittings in one-dimensional (1D) ballistic waveguides [29]. Both types of devices were fabricated by scanning probe lithography and the measurements were performed in a field oriented perpendicular to the heterostructure. Finally, an in-plane value of 1.5 was found for a gate-defined QD in an amorphous LAO/STO heterostructure [17].

Here, we systematically study the $g$-factors of spin states in gate-defined quantum dots in LAO/STO. We consider devices operating in the two overall regimes: those that exhibit effective repulsive electron interactions and single-electron tunneling — similar to conventional semiconductor QDs-and those that exhibit attractive interactions where transport and addition spectra are governed by electron pairs-being a special feature of the oxide system. We measure the anisotropy of the $g_{\mathrm{e}}$ tensor and relate it to the effective QD confinement. Finally, despite the large effective mass in LAO/STO, we observe both regimes of alternating ground-state spin filling according to the Pauli principles and of equal spin-filling sequences as expected for dominating exchange interactions. We discuss the implications for the oxide QD level structure and strength of electron-electron interactions using the wellstudied GaAs QDs as a reference.

In quantum dots, the $g$-factor can be extracted by measuring the addition spectrum in a magnetic field. Within the constant interaction model, the energy of the $N$ th charge state is given by $E(N)=\left(e N-C_{\mathrm{sg}} V_{\mathrm{sg}}\right)^{2} / 2 C_{\Sigma}+\epsilon_{N}$, where $\epsilon_{N}$ denotes the confinement energy, $C_{\Sigma}$ is the total capacitance, and $C_{\mathrm{sg}}$ is the capacitance to the gate electrode which is biased at $V_{\mathrm{sg}}$ and controls the occupation. This is schematically illustrated in Fig. 1(a) where black and orange lines correspond to even and odd $N$, respectively. At $V_{\mathrm{sg}}$ values corresponding to charge degeneracy, $E\left(N+1, V_{\mathrm{sg}}\right)=$ $E\left(N, V_{\mathrm{sg}}\right) \Rightarrow e \alpha V_{\mathrm{sg}}=E_{C} N+\delta_{N}$, sequential tunneling is allowed, resulting in Coulomb blockade (CB) conductance peaks [orange circles in Fig. 1(a)]. Here, $E_{C}=e^{2} / C_{\Sigma}, \alpha=$ $C_{\mathrm{sg}} / C_{\Sigma}$, and $\delta_{N}=\epsilon_{N+1}-\epsilon_{N}$ are the charging energy, lever arm, and field-dependent level spacings, respectively. Letting $s_{N}$ denote the component of the total spin along the direction of $B$ of the $N$ th charge state, the Zeeman effect shifts the energies $\epsilon_{N}(B)=\epsilon_{N}^{0}+s_{N} g_{\mathrm{e}} \mu_{\mathrm{B}} B$ as shown by the orange dashed curves in Fig. 1(a), and the separations of successive $C B$ peaks $\Delta V_{\mathrm{sg}}=\frac{1}{e \alpha}\left(s_{N+1}-2 s_{N}+s_{N-1}\right) g_{\mathrm{e}} \mu_{\mathrm{B}} B$ depend linearly on $B$ with a slope of $0, \pm g_{\mathrm{e}}$ depending on the spin-filling sequence. In the special case of LAO/STO, mesoscopic devices of two classes have been reported, exhibiting either conventional repulsive interactions - similar to semiconductors - or operating in a regime of effective attractive interactions which favor pairing of electrons $[15,17]$. For the latter case, the underlying mechanism of the attraction remains unknown, however, both the ground-state and excited-state energy spectra $[15,17]$ have been accurately accounted for by introducing effective negative charging energy $U$ in a single-orbital Anderson model. In Fig. 1(a) the odd- $N$ parabola (solid blue curve) then resides at energies above the even- $N$ states. In this case, the ground-state occupation remains even for all $V_{\mathrm{sg}}$, and at the even-even degeneracy points access to the odd- $N$ charge states requires an energy $|U|$. Thus, linear transport processes either occur by pair tunneling or thermally excited single-electron transport, which are both suppressed at low temperature and weak tunnel coupling, resulting in a transport gap for low bias $\left|e V_{\text {sd }}\right|<|U|$ $[16,17]$. Upon applying a magnetic field, the spin-degenerate odd- $N$ states are split by the Zeeman effect (dashed blue lines), and at the characteristic pairing field, $B_{\mathrm{p}}=|U| / g_{\mathrm{e}} \mu_{\mathrm{B}}$, 
the splitting exceeds $|U|$ and single-electron transport is restored. For $B>B_{\mathrm{p}}$, the situation becomes equivalent to the conventional $U>0$ case discussed above and the peak shifts can be used to determine $g_{\mathrm{e}}$ [blue circles in Fig. 1(a)]. We note that the spin-orbit correction to the $g$-factor from the bare value $g_{0}=2$ is negative but the QD measurement is insensitive to the sign and in the following $g_{\mathrm{e}}$ denotes the magnitude of the $g$-factor.

The fabrication of LAO/STO QDs followed the approach of Refs. [17,30]: A $\mathrm{TiO}_{2}$ terminated STO substrate was covered by $30 \mathrm{~nm}$ of LaSrMnO (LSMO) using pulsed laser deposition (PLD) to protect the STO surface during the subsequent lithography steps. Ti/Au $(5 \mathrm{~nm} / 45 \mathrm{~nm})$ gate structures were defined by $e$-beam lithography and selective etching of LSMO in $\mathrm{KI}(3 M): \mathrm{HCl}(37 \%): \mathrm{H}_{2} \mathrm{O}(2: 2: 35)$ exposed the STO surface in a mesa pattern centered on the gate structure. Finally, $10 \mathrm{~nm}$ of LAO was deposited by room-temperature PLD creating a conducting interface in the exposed region [31,32]. Figure 1(a) shows a scanning electron micrograph of a finished device with the gate design used for sample S1. The two gates define a QD connected to the source and drain reservoirs by narrow constrictions. For sample $\mathrm{S} 2$ the gates were in the form of two facing finger gates defining a single constriction where a QD effectively forms for $V_{\text {sg }}$ close to pinch-off due to local disorder-induced fluctuations in the potential [33,34]. The details of sample S2 are presented in Supplemental Fig. S1 [35] and Ref. [17]. In the following, we define the coordinate system such that $x(y)$ is in-plane parallel (perpendicular) to the current, $z$ is out of plane, and $\theta(\phi)$ denote the angle from $x$ to $z(y)$. Measurements were carried out in a dilution refrigerator with a base temperature $T=15 \mathrm{mK}$ and equipped with a 3D vector magnet capable of applying 6,1 , and $1 \mathrm{~T}$ in three orthogonal directions. The differential conductance $G$ was measured using conventional lock-in techniques with a $10-\mu \mathrm{V}$ ac excitation. From Hall measurements of the ungated mesa adjacent to the QD gates we found a low-temperature carrier density of $n_{s} \sim 2 \times 10^{13} \mathrm{~cm}^{-2}$, mobility $\mu \sim 250-600 \mathrm{~cm}^{2} / \mathrm{V} \mathrm{s}$, and a corresponding mean free path of $\sim 20-50 \mathrm{~nm}$ typical for the amorphous LAO/STO heterostructures under these PLD conditions $[17,36]$. Sample S1 exhibited conventional single-electron transport while S2 showed effective attractive interactions and electron pairing $[15,17]$.

Figure 1 presents the bias spectroscopy and magnetic field evolution of Coulomb peaks for the two devices: S1 [Figs. 1(b) and 1(c)] with $U>0$ and S2 [Figs. 1(d) and 1(e)] with $U<0$. Consider first $G$ vs $V_{\text {sd }}$ and $V_{\text {sg }}$ in Fig. 1(b). At low bias, $G$ is suppressed in diamond-shaped regions typical for a $\mathrm{QD}$ in the $\mathrm{CB}$ regime and the diamond size yields a lever arm $\alpha_{\mathrm{S} 1}=3.3 \pm 0.3 \times 10^{-2}$ and charging energy $E_{C} \approx$ $200 \mu \mathrm{eV}$. The uncertainty in $\alpha$ is caused by the smearing in Fig. 1(b) which makes the identification of the diamond height uncertain. Since $\alpha$ converts $V_{\text {sg }}$ shifts to energy, this propagates into a $\sim 10 \%$ uncertainty for the $g$-factor values extracted in the following. Figure 1(c) shows the evolution of the zero-bias CB peaks in a perpendicular magnetic field $B_{\mathrm{z}}$. For $B_{\mathrm{z}} \lesssim 2 \mathrm{~T}$, the peak positions shift linearly with $B_{\mathrm{z}}$ to alternating higher/lower $V_{\mathrm{sg}}$, consistent with the description above and previous reports for semiconductor QDs [2]. At higher fields the regular pattern is interrupted due to level crossing.
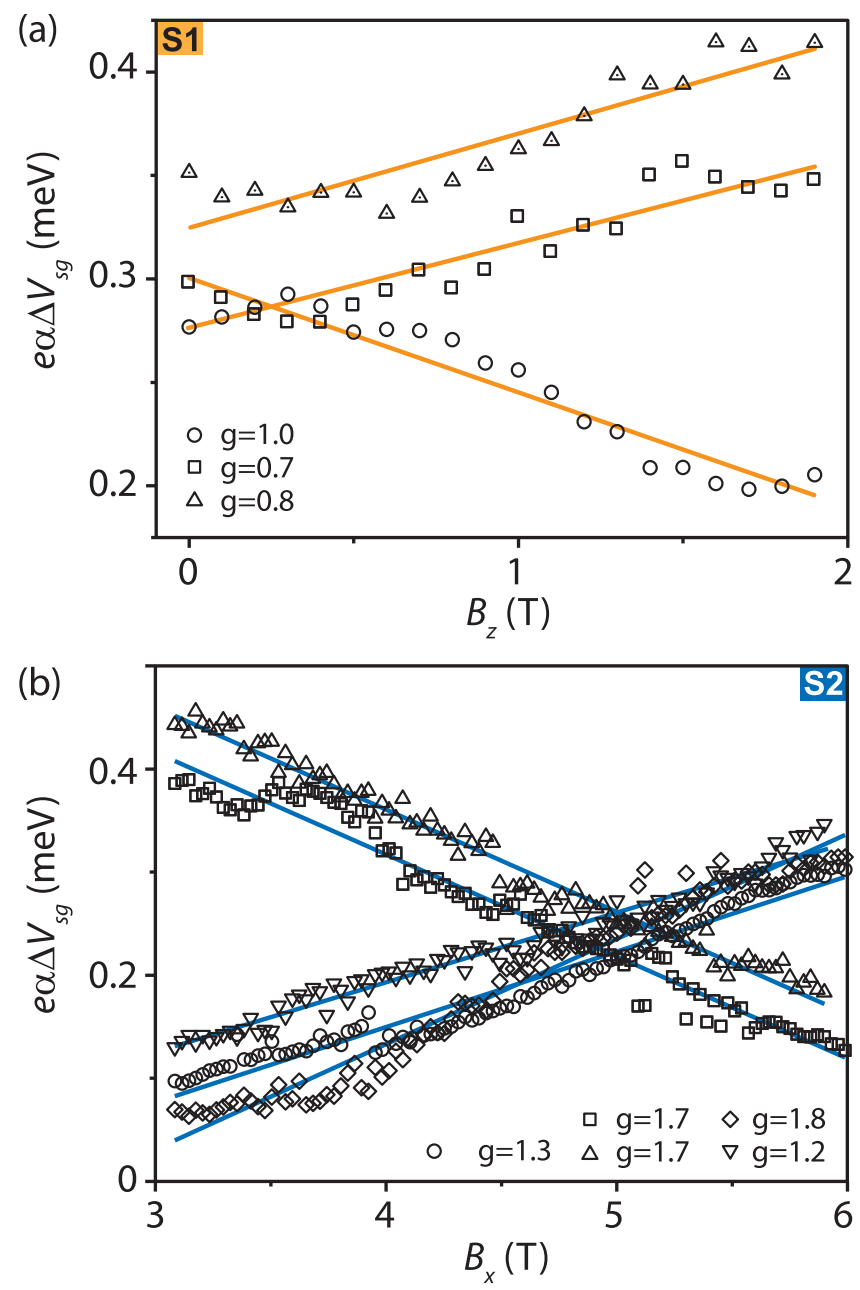

FIG. 2. Field dependence of the $V_{\mathrm{sg}}$ separation of zero-bias CB peaks. The values have been converted into energy by scaling with the lever $\operatorname{arm} \alpha$. (a) and (b) show results for devices S1 and S2 with effective $U>0$ and $U<0$, respectively, and symbols correspond to those in Figs. 1(c) and 1(e). The corresponding $g$-factors are found from linear fits (solid lines).

Corresponding measurements for sample S2 are shown in Figs. 1(d) and 1(e). Again, diamond-shaped regions of low conductance are observed, however, with a finite gap for $\left|e V_{\text {sd }}\right|<|U|=100-160 \mu \mathrm{eV}$. The magnetic field dependence of $G$ vs $V_{\text {sg }}$ in Fig. 1(e) shows that zero-bias peaks are restored at the pairing field $1 \lesssim B_{\mathrm{p}} \lesssim 2 \mathrm{~T}$. Further increasing $B$ causes linear peaks to split, consistent with the schematic picture in Fig. 1(a). Thus S2 is dominated by an attractive interaction and electron pairing at low field, and the Coulomb diamonds in Fig. 1(d) correspond to the addition of the double charge yielding $\alpha_{\mathrm{S} 2}=(5.5 \pm 0.9) \times 10^{-3}$ and $E_{C} \approx 250 \mu \mathrm{eV}$.

For both $\mathrm{S} 1$ and $\mathrm{S} 2$, a sequence of alternating dispersions in magnetic fields is seen, as expected in a QD where the singleparticle levels are successively filled by electrons of opposite spins according to the Pauli principle. To find $g_{\mathrm{e}}$, each peak $G\left(V_{\mathrm{sg}}\right)$ was fitted to a Lorentzian line shape, and the separations of peak center positions $\Delta V_{\mathrm{sg}}(B)$ are shown in Figs. 2(a) and 2(b) for the two cases. The corresponding values of $g_{\mathrm{e}}$ are 
extracted from the slope of linear fits (solid curves) and stated on the figure. For each device, the values show only a small spread between charge states and are lower than the bare value $g_{0}=2$. In general, $g_{\mathrm{e}}$ is affected by the strength of spin-orbit coupling, the magnitude of the orbital magnetic moments, and the level spacing of the dot. The latter two depend on the effective size of the QD and on the individual wave function. Since $g_{\mathrm{e}} \neq g_{0}$ and differs between the two devices, we conclude that both the SOI and the geometry play an important role. The effective size of the QD is related to the capacitance/charging energy, however, a size estimate is complicated by the electric field dependence of the dielectric constant of STO. This problem was numerically treated in Ref. [12] and for $E_{C} \approx$ $200-300 \mu \mathrm{eV}$ a very small dot radius of $\sim 10 \mathrm{~nm}$ is estimated, comparable to the out-of-plane heterostructure confinement. For such tight isotropic confinement we expect efficient quenching of the orbital moment consistent with the measured $g_{\mathrm{e}} \lesssim 2$ in Fig. 2 and $g_{\mathrm{e}} \approx 1.9$ measured with the field oriented in the orthogonal out-of-plane direction for S2 (see Supplemental Material [35]). Note that the latter measurement required a thermal cycle and retuning of the device.

For larger $V_{\mathrm{sg}}$, the occupation of the QD increases and the weaker electrostatic confinement leads to a stronger coupling of the QD to the leads, and we expect an increase of the electrostatic size. In this case, the magnitude of $g_{\mathrm{e}}$ may be increased and anisotropies may result due to increased orbital contributions. Figure 3(a) shows the bias spectroscopy of S2 in this regime. The diamond structure is still apparent, but the charging energy is reduced to $E_{C}=25-50 \mu \mathrm{eV}$, peaks are broadened due to the stronger coupling and larger $\mathrm{QD}$, and the pairing gap around zero bias is replaced by a lowbias resonance previously associated with the charge Kondo effect [17].

Upon applying a magnetic field, the bifurcation of the resonances as the Zeeman energy exceeds $|U|$ is shown in Fig. 3(b) for three different field orientations. $B_{\mathrm{p}}$ is reduced compared to the more closed regime $[15,16]$, and at these $V_{\mathrm{sg}}, B_{\mathrm{p}}$ is within reach of our vector magnet, thus allowing us to study the anisotropy of $g_{\mathrm{e}}$ of the same charge state. For directions with low $g_{\mathrm{e}}$, only a small field range exceeding $B_{\mathrm{p}}$ is available, and $g_{\mathrm{e}}$ is most accurately determined from the pairing field $B_{\mathrm{p}}=|U| / g_{\mathrm{e}} \mu_{\mathrm{B}}$. The value for $|U|=65 \pm 15 \mu \mathrm{eV}$ is estimated as the average value of $g_{\mathrm{e}} \mu_{\mathrm{B}} B_{\mathrm{p}}$ for all orientations and $g_{\mathrm{e}}$ estimated from the slope of splitting for $B_{\mathrm{p}}<B<1 \mathrm{~T}$. From Fig. 3(b), $B_{\mathrm{p}}$ was found by fitting a double-Lorentzian profile to the $G\left(V_{\mathrm{sg}}\right)$ traces at each $B$ and identifying $B_{\mathrm{p}}$ as the field where the peak separations extrapolate to zero. Figure 3(c) shows the result for measurements with $B$ oriented in the $x y, y z$, and $x z$ planes, and Fig. 3(d) shows the corresponding results for $g_{\mathrm{e}}=|U| / B_{\mathrm{p}} \mu_{\mathrm{B}}$. A systematic anisotropy is evident, with $g_{\mathrm{e}}$ being larger, i.e. smaller $B_{\mathrm{p}}$, by a factor of $\approx 3$ in the out-of-plane direction compared to the in-plane directions [Fig. 3(d), leftmost panel] in which $g_{\mathrm{e}}$ is nearly isotropic. The anisotropic $g$-factor can be written in the general form [37] $g_{\mathrm{e}}(B)=\sqrt{g_{1}^{2} B_{1}^{2}+g_{2}^{2} B_{2}^{2}+g_{3}^{2} B_{3}^{2}} / B$ where $g_{1}, g_{2}, g_{3}$ and $B_{1}, B_{2}, B_{3}$ are the components of $g_{\mathrm{e}}$ and $B$ along three orthogonal principal axes. From the device design and the apparent symmetry observed in Figs. 3(c) and 3(d) the device axis ( $x$, $y$, and $z$ ) are natural candidates for the principal axes $\left[g_{1}=\right.$
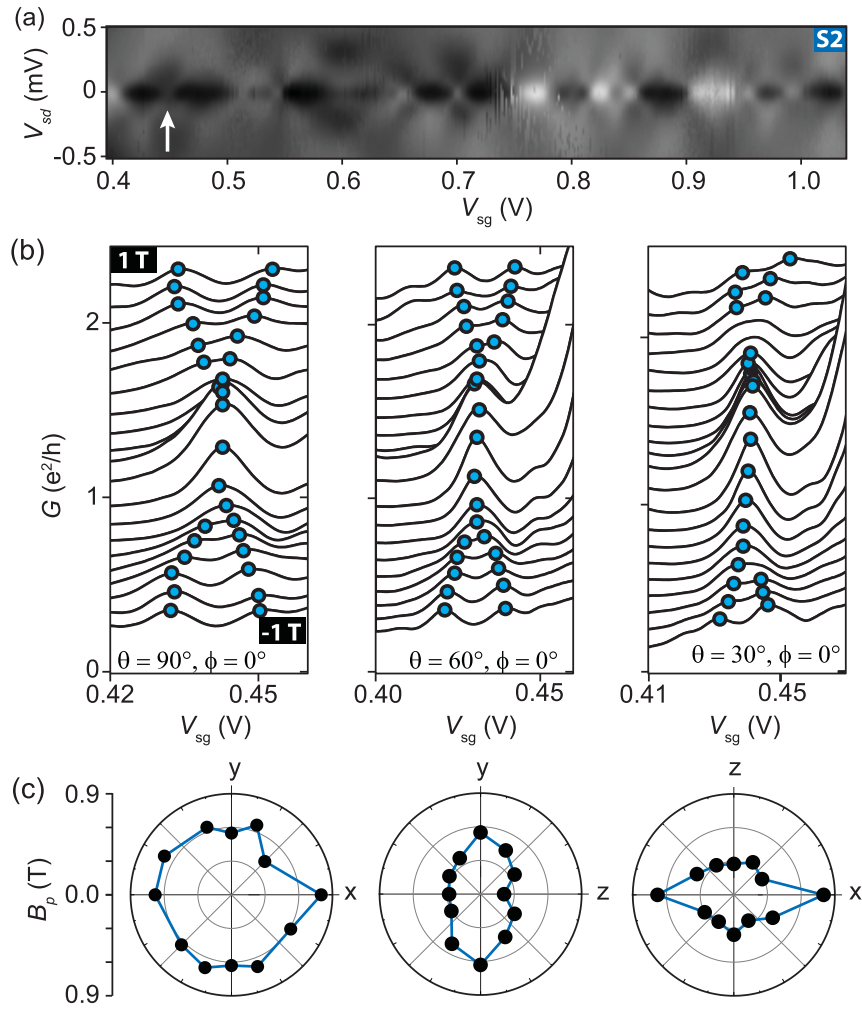

(d)
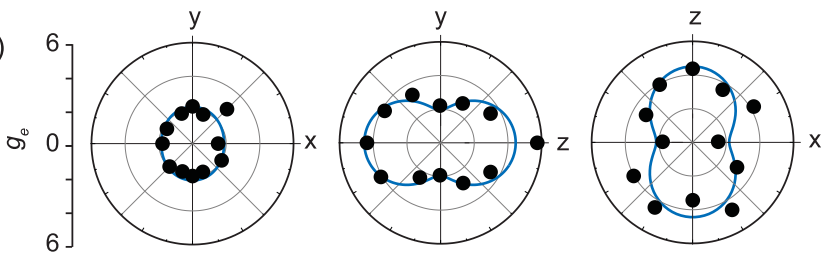

FIG. 3. (a) Bias spectroscopy of sample S2 in a regime of higher $V_{\mathrm{sg}}$. (b) Zero-bias conductance vs $V_{\mathrm{sg}}$ for equidistant values of magnetic field magnitude from -1 to $1 \mathrm{~T}$; curves are offset by $(B+1) e^{2} / h$ with $B$ in tesla. The three panels show measurements for different orientations of $B$ as indicated. For each trace, the peak positions indicated by markers are found by fitting the trace to a single- or double-peak profile and the pairing field is identified. (d)-(f) Anisotropy of $g_{e}$ extracted from $B_{\mathrm{p}}$ (see text).

$\left.g_{\mathrm{x}}, g_{2}=g_{\mathrm{y}}, g_{3}=g_{\mathrm{z}}\right]$. The solid lines in Fig. 3(d) show fits to this relation with $\left(g_{\mathrm{x}}, g_{\mathrm{y}}, g_{\mathrm{z}}\right)=(1.9,2.2,4.5)$ providing a good description of the data. Since the SOI-induced correction from $g_{0}=+2$ to the $g$-factor is always negative, the measured out-of-plane value $g_{\mathrm{e}}=\left|g_{\mathrm{e}}\right|=4.5$ shows that in this case the $g$-factor is negative, and as it remains finite for all angles in Fig. 3 this must be the case for all directions. The observed anisotropy is consistent with an approximate in-plane symmetric disk-shaped QD defined in the oxide heterostructure, where the angular magnetic moment will naturally be oriented normal to the 2DEG. In this case we expect $g_{\mathrm{x}} \approx g_{\mathrm{y}}$ and a spin-orbit enhanced perpendicular $g_{\mathrm{z}}$. Our value is consistent with $g_{\mathrm{z}}=5$ found in a recent high-field study of $\mathrm{SdH}$ oscillations in bulk LAO/STO [25]. For a disk-shaped QD of diameter $D$ and height $H$ the aspect ratio is related to the orbital moments [28] $D / H=\mu_{\mathrm{z}}^{\text {orb }} / \mu_{\|}^{\text {orb }}$ which can be estimated from $g_{\mathrm{e}}$ since $g_{x, y, z}=2\left(\mu_{x, y, z}^{\mathrm{spin}}+\mu_{x, y, z}^{\mathrm{orb}}\right) / \mu_{\mathrm{B}}$ where 

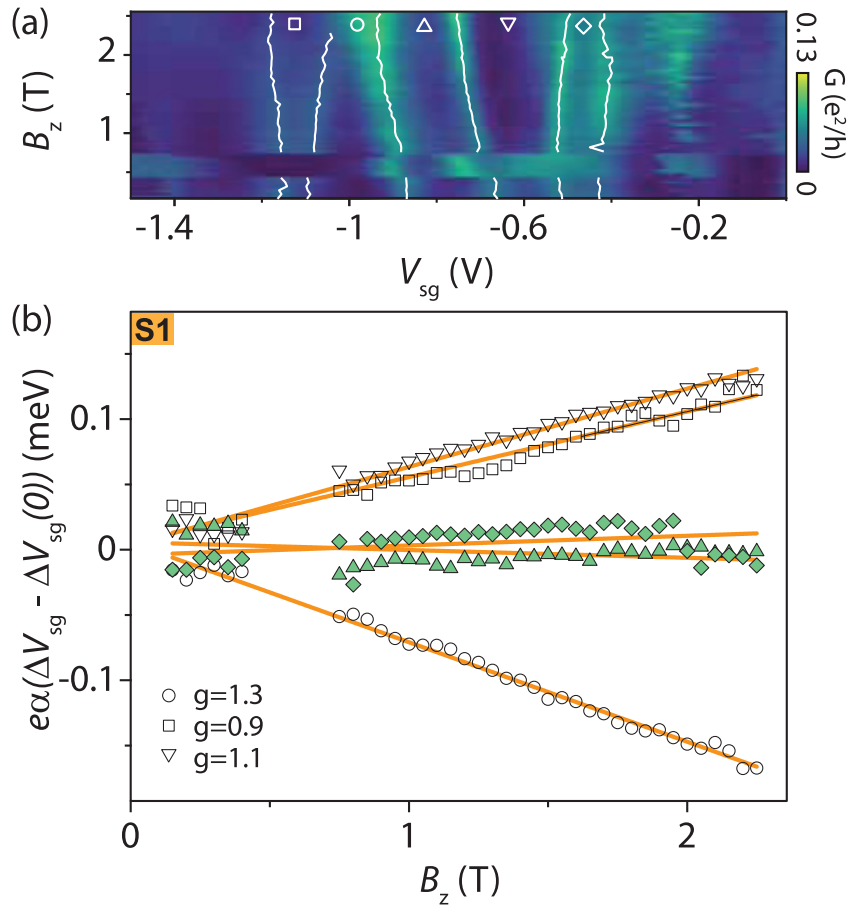

FIG. 4. (a) Zero-bias conductance vs $V_{\mathrm{sg}}$ and $B_{\mathrm{z}}$ for sample $\mathrm{S} 1$ in a different gate regime. (b) The field dependence of the peak separations offset to coincide at $B=0$. Solid lines show linear fits and the corresponding $g$-factors are indicated. Field-independent peak spacings (solid symbols) result from the successive addition of electrons to the QD with the same spin.

$\mu^{\text {spin }} \approx \mu_{\mathrm{B}}$. Taking the average $g_{\|} \sim\left(g_{\mathrm{x}}+g_{\mathrm{y}}\right) / 2=2.1$ as the in-plane $g$-factor we get $D / H=\left(g_{\mathrm{z}}-2\right) /\left(g_{\|}-2\right) \approx 1.6$. For planar LAO/STO the 2DEG thickness depends on the density, and values $\sim 10-30 \mathrm{~nm}$ increasing with the gate were recently reported [38]. Using this for $H$ then yields $D \sim 16-48 \mathrm{~nm}$. Even with the largest thickness_-possibly relevant for the QD at the largest $V_{\mathrm{sg}}$ - this value is smaller than an estimate based on the charging energy and the electrostatic model of Ref. [12] which yields $D \sim 100 \mathrm{~nm}$. The reason for the discrepancy is unknown, however, we note that the electrostatic model assumes a 2DEG thickness of $10 \mathrm{~nm}$ and may thus overestimate $D$. Future studies of LAO/STO QD devices with systematically varying gate geometries and continuously tracking the change of $E_{C}$ and $g_{\mathrm{e}}$ with $V_{\mathrm{sg}}$ [39] could shed light on the link between these two complementary probes of the effective QD size and shape.

So far we have focused on measurements exhibiting alternating spin filling according to the Pauli principle. Figure 4(a) shows $G$ vs $V_{\text {sg }}$ and $B_{\mathrm{z}}$ for sample $\mathrm{S} 1$ in a different gate range and Fig. 4(b) shows the corresponding field dependence of the peak separations. In contrast to Fig. 2, three branches are now observed: Two repeat the linear increasing/decreasing behavior expected for a ground-state spin difference of $1 / 2$ and a $g$-factor consistent with the values from Fig. 2, and a third field-independent branch (solid symbols) indicates the successive addition of electrons of the same spin. The latter case is expected when exchange interactions dominate and favor parallel spin alignment according to Hund's rule [40,41]. The two regimes are distinguished by the ratio of the level spacing $\delta \sim \hbar^{2} / m^{*} A$ ( $A$ being the QD area) and the Coulomb interaction $U_{e e} \sim e^{2} / \epsilon * r_{e e}$, where $r_{e e}$ is the typical electron-electron separation [42]. As a reference, we consider GaAs [40,41] and graphene [42] QDs of similar sizes. There, the exchange interaction tends to dominate, but spin pairs are occasionally observed, suggesting that $\delta \lesssim U_{e e}$. The large effective mass of STO compared to GaAs [43], $m_{\mathrm{STO}}^{*}=0.5-2.7 m_{\mathrm{e}} \gg m_{\mathrm{GaAs}}^{*}=$ $0.067 m_{\mathrm{e}}$, leads to a significantly smaller level spacing of STO, $\delta \epsilon_{\mathrm{GaAs}} / \delta \epsilon_{\mathrm{STO}}=7-40$. However, the larger (field-dependent) dielectric constant $\epsilon_{\mathrm{STO}}=2-20 \times 10^{3} \gg \epsilon_{\mathrm{GaAs}}=12.9$ reduces the interactions by a factor $U_{e e}^{\mathrm{GaAs}} / U_{e e}^{\mathrm{STO}} \sim 1.5-15 \times$ $10^{2}$. Thus, the exchange interaction in LAO/STO QDs is expected to be less important compared to GaAs QDs of similar size. However, it may still play a role, when $\epsilon_{\mathrm{STO}}$ is reduced by the field from local gates, as indeed expected for the LAO/STO QDs [12]. These rough estimates appear consistent with the observations of both regimes in our measurements.

In conclusion, we have fabricated gate-defined quantum dot devices from LAO/STO oxide heterostructures and extracted the effective $g$-factor and its anisotropy from the low-temperature addition spectrum in a magnetic field. Devices in two regimes were measured: those dominated by single electron tunneling, and those dominated by attractive interactions and electron pairing at low field. We find no significant differences in the $g$-tensors in the two cases. In the low- $V_{g}$ regime closest to pinch-off, where the QD has the smallest spatial extent (consistent with the QD energy scales which suggest a small lateral extent similar to the heterostructure confinement), we find an isotropic $g$-tensor. At higher gate voltages, a clear anisotropy is found with the outof-plane component of the $g$-tensor being double that of the in-plane values. This is consistent with an out-of-plane orbital magnetic moment and a disk-shaped anisotropic geometry. Finally, we reported regimes where the successive addition of electrons of the same spin demonstrate the importance of exchange interactions in LAO/STO despite the large dielectric constant of STO. Thus, despite the very different energy scales and parameters of oxide heterostructures, the spin properties encoded in the $g$-factors are similar to semiconductor-based devices. Future experiments tracking the evolution of $g$-factor anisotropies in $V_{g}$ while tuning through the Lifshitz transition of the underlying band structure [23] could act as a tool for identifying the band character of the QD states. Noting that superconductivity in LAO/STO heterostructures is linked to the Lifshitz transition, we propose that such measurements may shed light on the origin of the attractive interactions leading to a negative $U$ in the QDs and the relation to superconductivity.

This work was supported by a research grant (00013157) from Villum Fonden. A.V.B., D.J.C., G.E.D.K.P., and M.v.S. conducted the sample fabrication, measurements, and data analysis. Y.G. and Y.C. conducted the sample growth. D.J.C., N.P., J.P., and T.S.J. supervised the project and wrote the paper with input from all authors. 
[1] D. Loss and D. P. DiVincenzo, Phys. Rev. A 57, 120 (1998).

[2] R. Hanson, L. P. Kouwenhoven, J. R. Petta, S. Tarucha, and L. M. K. Vandersypen, Rev. Mod. Phys. 79, 1217 (2007).

[3] J. Elzerman, R. Hanson, L. van Beveren, B. Witkamp, L. Vandersypen, and L. Kouwenhoven, Nature (London) 430, 431 (2004).

[4] F. H. L. Koppens, C. Buizert, K. J. Tielrooij, I. T. Vink, K. C. Nowack, T. Meunier, L. P. Kouwenhoven, and L. M. K. Vandersypen, Nature (London) 442, 766 (2006).

[5] M. Pioro-Ladriere, T. Obata, Y. Tokura, Y. S. Shin, T. Kubo, K. Yoshida, T. Taniyama, and S. Tarucha, Nat. Phys. 4, 776 (2008).

[6] K. C. Nowack, F. H. L. Koppens, Y. V. Nazarov, and L. M. K. Vandersypen, Science 318, 1430 (2007).

[7] S. Nadj-Perge, S. M. Frolov, E. P. A. M. Bakkers, and L. P. Kouwenhoven, Nature (London) 468, 1084 (2010).

[8] A. Ohtomo and H. Hwang, Nature (London) 427, 423 (2004).

[9] S. Thiel, G. Hammerl, A. Schmehl, C. W. Schneider, and J. Mannhart, Science 313, 1942 (2006).

[10] A. D. Caviglia, S. Gariglio, N. Reyren, D. Jaccard, T. Schneider, M. Gabay, S. Thiel, G. Hammerl, J. Mannhart, and J. M. Triscone, Nature (London) 456, 624 (2008).

[11] P. Gallagher, M. Lee, J. Williams, and D. Goldhaber-Gordon, Nat. Phys. 10, 748 (2014).

[12] H. Thierschmann, E. Mulazimoglu, N. Manca, S. Goswami, T. Klapwijk, and A. Caviglia, Nat. Commun. 9, 2276 (2018).

[13] A. Ron and Y. Dagan, Phys. Rev. Lett. 112, 136801 (2014).

[14] M. Tomczyk, G. Cheng, H. Lee, S. Lu, A. Annadi, J. P. Veazey, M. Huang, P. Irvin, S. Ryu, C.-B. Eom, and J. Levy, Phys. Rev. Lett. 117, 096801 (2016).

[15] G. Cheng, M. Tomczyk, S. Lu, J. P. Veazey, M. Huang, P. Irvin, S. Ryu, H. Lee, C.-B. Eom, C. S. Hellberg, and J. Levy, Nature (London) 521, 196 (2015).

[16] G. Cheng, M. Tomczyk, A. B. Tacla, H. Lee, S. Lu, J. P. Veazey, M. Huang, P. Irvin, S. Ryu, C.-B. Eom, A. Daley, D. Pekker, and J. Levy, Phys. Rev. X 6, 041042 (2016).

[17] G. E. D. K. Prawiroatmodjo, M. Leijnse, F. Trier, Y. Chen, D. V. Christensen, M. von Soosten, N. Pryds, and T. S. Jespersen, Nat. Commun. 8, 395 (2017).

[18] E. Maniv, A. Ron, M. Goldstein, A. Palevski, and Y. Dagan, Phys. Rev. B 94, 045120 (2016).

[19] A. Brinkman, M. Huijben, M. Van Zalk, J. Huijben, U. Zeitler, J. C. Maan, W. G. Van der Wiel, G. Rijnders, D. H. A. Blank, and H. Hilgenkamp, Nat. Mater. 6, 493 (2007).

[20] N. Reyren, S. Thiel, A. D. Caviglia, L. F. Kourkoutis, G. Hammerl, C. Richter, C. W. Schneider, T. Kopp, A.-S. Ruetschi, D. Jaccard, M. Gabay, D. A. Muller, J.-M. Triscone, and J. Mannhart, Science 317, 1196 (2007).

[21] L. Roth, B. Laz, and S. Zwerdling, Phys. Rev. 114, 90 (1959).

[22] Y. Kim, R. M. Lutchyn, and C. Nayak, Phys. Rev. B 87, 245121 (2013).
[23] A. Joshua, S. Pecker, J. Ruhman, E. Altman, and S. Ilani, Nat. Commun. 3, 1129 (2012).

[24] A. D. Caviglia, M. Gabay, S. Gariglio, N. Reyren, C. Cancellieri, and J. M. Triscone, Phys. Rev. Lett. 104, 126803 (2010).

[25] M. Yang, K. Han, O. Torresin, M. Pierre, S. Zeng, Z. Huang, T. V. Venkatesan, M. Goiran, J. M. D. Coey, Ariando, and W. Escoffier, Appl. Phys. Lett. 109, 122106 (2016).

[26] C. E. Pryor and M. E. Flatté, Phys. Rev. Lett. 96, 026804 (2006).

[27] J. van Bree, A. Y. Silov, P. M. Koenraad, and M. E. Flatte, Phys. Rev. Lett. 112, 187201 (2014).

[28] J. van Bree, A. Y. Silov, M. L. van Maasakkers, C. E. Pryor, M. E. Flatte, and P. M. Koenraad, Phys. Rev. B 93, 035311 (2016).

[29] A. Annadi, G. Cheng, H. Lee, J.-W. Lee, S. Lu, A. Tylan-Tyler, M. Briggeman, M. Tomczyk, M. Huang, D. Pekker, C.-B. Eom, P. Irvin, and J. Levy, Nano Lett. 18, 4473 (2018).

[30] F. Trier, G. E. D. K. Prawiroatmodjo, M. von Soosten, D. V. Christensen, T. S. Jespersen, Y. Z. Chen, and N. Pryds, Appl. Phys. Lett. 107, 191604 (2015).

[31] Y. Chen, N. Pryds, K. J, G. Koster, J. Sun, E. Stamate, B. Shen, G. Rijnders, and S. Linderoth, Nano Lett. 11, 3774 (2011).

[32] Z. Q. Liu, C. J. Li, W. M. Lu, X. H. Huang, Z. Huang, S. W. Zeng, X. P. Qiu, L. S. Huang, A. Annadi, J. S. Chen, J. M. D. Coey, T. Venkatesan, and Ariando, Phys. Rev. X 3, 021010 (2013).

[33] J. A. Nixon and J. H. Davies, Phys. Rev. B 41, 7929 (1990).

[34] J. H. F. Scott-Thomas, S. B. Field, M. A. Kastner, H. I. Smith, and D. A. Antoniadis, Phys. Rev. Lett. 62, 583 (1989).

[35] See Supplemental Material at http://link.aps.org/supplemental/ 10.1103/PhysRevMaterials.4.122001 for additional data and description of devices.

[36] G. E. D. K. Prawiroatmodjo, F. Trier, D. V. Christensen, Y. Chen, N. Pryds, and T. S. Jespersen, Phys. Rev. B 93, 184504 (2016).

[37] M. D. Schroer, K. D. Petersson, M. Jung, and J. R. Petta, Phys. Rev. Lett. 107, 176811 (2011).

[38] D. Li, S. Lemal, S. Gariglio, Z. Wu, A. Fete, M. Boselli, P. Ghosez, and J.-M. Triscone, Adv. Sci. 5, 1800242 (2018).

[39] S. Csonka, L. Hofstetter, F. Freitag, S. Oberholzer, C. Schoenenberger, T. S. Jespersen, M. Aagesen, and J. Nygard, Nano. Lett. 8, 3932 (2008).

[40] S. Lindemann, T. Ihn, T. Heinzel, W. Zwerger, K. Ensslin, K. Maranowski, and A. C. Gossard, Phys. Rev. B 66, 195314 (2002).

[41] J. Folk, C. Marcus, R. Berkovits, I. Kurland, I. Aleiner, and B. Altshuler, Phys. Scr., T 90, 26 (2001).

[42] J. Güttinger, T. Frey, C. Stampfer, T. Ihn, and K. Ensslin, Phys. Rev. Lett. 105, 116801 (2010).

[43] Y.-Y. Pai, A. Tylan-Tyler, P. Irvin, and J. Levy, Rep. Prog. Phys. 81, 036503 (2018). 